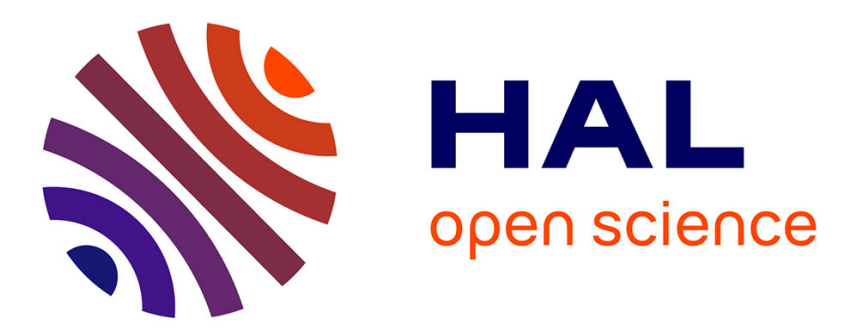

\title{
Inhibition, Enhancement, and Control of Spontaneous Emission in Photonic Nanowires
}

Joël Bleuse, Julien Claudon, M. Creasey, N.S. Malik, Jean-Michel Gérard, Ivan Maksymov, Jean-Paul Hugonin, Philippe Lalanne

\section{To cite this version:}

Joël Bleuse, Julien Claudon, M. Creasey, N.S. Malik, Jean-Michel Gérard, et al.. Inhibition, Enhancement, and Control of Spontaneous Emission in Photonic Nanowires. Physical Review Letters, 2011, 106 (10), pp.103601. 10.1103/PhysRevLett.106.103601 . hal-00680596

\section{HAL Id: hal-00680596 \\ https://hal-iogs.archives-ouvertes.fr/hal-00680596}

Submitted on 1 Dec 2015

HAL is a multi-disciplinary open access archive for the deposit and dissemination of scientific research documents, whether they are published or not. The documents may come from teaching and research institutions in France or abroad, or from public or private research centers.
L'archive ouverte pluridisciplinaire HAL, est destinée au dépôt et à la diffusion de documents scientifiques de niveau recherche, publiés ou non, émanant des établissements d'enseignement et de recherche français ou étrangers, des laboratoires publics ou privés. 


\title{
Inhibition, Enhancement, and Control of Spontaneous Emission in Photonic Nanowires
}

\author{
Joël Bleuse, ${ }^{*}$ Julien Claudon, ${ }^{\dagger}$ Megan Creasey, Nitin. S. Malik, and Jean-Michel Gérard \\ CEA-CNRS-UJF group “Nanophysique et Semiconducteurs," CEA, INAC, SP2M, F-38054 Grenoble, France \\ Ivan Maksymov, Jean-Paul Hugonin, and Philippe Lalanne \\ Laboratoire Charles Fabry de l'Institut d'Optique, CNRS, Université Paris-Sud, \\ Campus Polytechnique, RD 128, 91127 Palaiseau, France \\ (Received 13 October 2010; revised manuscript received 23 December 2010; published 11 March 2011)
}

\begin{abstract}
We experimentally investigate the spontaneous emission (SE) rates of single InAs quantum dots embedded in GaAs photonic nanowires. For a diameter leading to the optimal confinement of the fundamental guided mode $\mathrm{HE}_{11}$, the coupling to $\mathrm{HE}_{11}$ dominates the SE process and an increase of the $\mathrm{SE}$ rate by a factor of 1.5 is achieved. When the diameter is decreased, the coupling to this mode vanishes rapidly, thus allowing the coupling to the other radiation modes to be probed. In these conditions, a SE inhibition factor of 16, equivalent to the one obtained in state-of-the-art photonic crystals, is measured. These results, which are supported by fully vectorial calculations, confirm the potential of photonic nanowires for a nearly perfect, broadband SE control.
\end{abstract}

DOI: 10.1103/PhysRevLett.106.103601

PACS numbers: 42.50.- p, 78.55.Cr, 78.67.Hc

Funneling a large fraction $\beta$ of the spontaneous emission (SE) of an emitter into a single optical mode has long been thought of as a powerful strategy for improving the performance of optoelectronic devices, such as microlasers or single-photon sources [1]. A first route to achieve this ambitious goal consists of enhancing the vacuum fluctuations associated with the mode of interest, so as to selectively increase the SE rate into this mode. In the solid state, this approach was first demonstrated by using the Purcell effect that arises when an emitter is inserted into a resonant optical microcavity [2]. In a seminal paper, Yablonovitch proposed instead to suppress the SE into the "useless" optical modes by using a photonic band gap structure [3]. This approach is demanding, from both the conception and fabrication points of view, because it requires a control over a continuum of modes. Inhibition in the optical domain has been demonstrated in 3D-photonic materials [4]; however, the highest inhibition factor reported so far $(\sim 17)$ was measured in the more mature technology of 2D-photonic crystals [5]. Recent experiments have explored the nonresonant SE control provided by a waveguide defined in such a structure [6,7]. Of course, SE enhancement and inhibition can be combined, as in a gold-coated micropillar cavity [8] or in 2D-photonic crystals with a point defect [9].

In this rich landscape, photonic nanowires are relatively simple dielectric structures for which a broadband and efficient SE control was predicted [10-13]. These monomode waveguides, made of a high refractive index material, ensure a tight lateral confinement of the guided mode and simultaneously provide a strong screening of the other modes. Experimentally, they were first introduced in a pioneering work on high- $\beta$ ring microlasers integrated on a chip [10]. Very recently, a vertical geometry, optimizing both the $\beta$ factor and the light collection in the far field, was introduced [13] to realize a highly efficient singlephoton source $[14,15]$. However, an experimental study of the SE dynamics in these systems or any direct evidence of SE inhibition is still lacking.

In this Letter, we use time-resolved photoluminescence experiments to investigate the SE of a single InAs quantum $\operatorname{dot}(\mathrm{QD})$ inserted in a cylindrical GaAs photonic nanowire. Varying the wire diameter dramatically changes the SE rate into the fundamental guided mode by several orders of magnitude. When this mode is deconfined, the total SE rate is dominated by the coupling to the continuum of radiative modes. A strong SE inhibition, comparable to state-of-theart photonic band gap structures, is then directly evidenced. When the mode is optimally confined, a significant SE enhancement demonstrates the good coupling of the emitter to the guided mode. These results that are supported by 3D fully vectorial computational results [16] confirm the potential of photonic nanowires to funnel nearly all the SE of an emitter into a single mode.

We first consider an infinitely long photonic nanowire with a cylindrical section of diameter $d$. It is made of GaAs $(n=3.45)$ and is surrounded by a low-index air cladding $\left(n_{c}=1\right)$. The structure always supports a fundamental guided mode $\mathrm{HE}_{11}$ whose group index is denoted $n_{g}$. The mode lateral confinement is quantitatively described by the effective surface area $S_{\text {eff }}=\int n(\mathbf{r})^{2}|\mathbf{E}(\mathbf{r})|^{2} d^{2} \mathbf{r} / \max$ $\left[n(\mathbf{r})^{2}|\mathbf{E}(\mathbf{r})|^{2}\right]$. The maximum SE rate into $\mathrm{HE}_{11}$ is obtained for an emitter (supposedly pointlike, with a vacuum wavelength $\lambda$ ) having an in-plane dipole and located on the wire axis:

$$
\bar{\Gamma}_{m}=2 \times \frac{3}{8 \pi} \frac{(\lambda / n)^{2}}{S_{\text {eff }}} \frac{n_{g}}{n} .
$$


The bar indicates a normalization to $\Gamma_{0}$, the SE rate in bulk GaAs, and the factor of 2 accounts for the contribution of the 2 counterpropagating $\mathrm{HE}_{11}$ modes supported by the structure. This Purcell factor depends only on the waveguide properties and constitutes a figure of merit of the SE enhancement in a waveguide. It is plotted in Fig. 1 against the reduced wire diameter $d / \lambda$. For $d / \lambda=0.235$, an optimal transverse confinement of the mode associated with an increased group index $\left[S_{\text {eff }}=0.41 \times(\lambda / n)^{2}\right.$ and $n_{g}=$ $1.55 \times n]$ results in an optimal emission rate $\bar{\Gamma}_{m}=0.9$, close to the bulk value.

The emitter is also coupled to a continuum of so-called radiative modes and possibly to other guided modes, with a total rate $\bar{\gamma}$. The mode $\mathrm{TE}_{01}$, with a zero electrical field on the wire axis, is guided by the structure for $d / \lambda>0.230$. After $\mathrm{HE}_{11}, \mathrm{HE}_{21}$ is the second mode with a nonzero field on the wire axis; it is present for $d / \lambda>0.338$. In the very small diameter limit $(d / \lambda \ll 1)$ the coupling to the radiative modes can be treated analytically and is found to be independent of the emitter location [17]: $\bar{\gamma}=4 /\left[n\left(n^{2}+\right.\right.$ $\left.1)^{2}\right]$. In a structure made of a high index material, the $n^{-5}$ scaling indicates a very efficient screening of the radiative modes that leads to a huge inhibition of the SE into these modes $(\bar{\gamma}=1 / 144$ for GaAs). Quite remarkably, this large effect has not been observed before. To be complete, the dipole orientation plays here a crucial role: If the dipole is parallel to the wire axis, $\bar{\gamma}=n^{-1}$ is much larger.

Photonic nanowires with a larger diameter also exhibit a significant screening of the radiative modes. However, the simple picture of the previous paragraph is no longer valid: Both the magnitude and the spatial dependence of $\bar{\gamma}$ are modified. To shed light on this important property, we first numerically investigate the reciprocal problem, namely, the screening of a single plane wave whose wave vector and polarization are perpendicular to the wire axis. The corresponding field maps are presented in Fig. 2 for three critical diameters. When $d / \lambda$ is increased from 0.05 to 0.19 , the field amplitude inside the structure increases progressively but remains approximately uniform. When

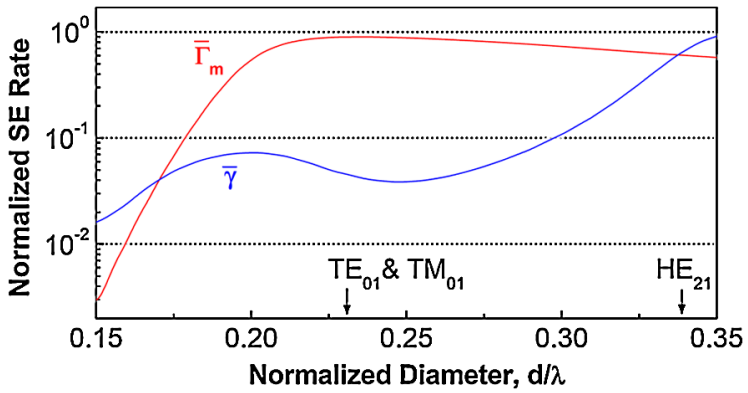

FIG. 1 (color online). Normalized $\mathrm{SE}$ rates into the $\mathrm{HE}_{11}$ modes, $\bar{\Gamma}_{m}$, and into the other modes, $\bar{\gamma}$, as a function of $d / \lambda$. The emitter, with an in-plane dipole, is located on the axis of an infinitely long photonic nanowire. The arrows indicate the cutoff of higher order guided modes. $d / \lambda$ exceeds 0.19 , the field amplitude becomes dependent on the position inside the wire and the screening effect is maximum in the vicinity of the wire axis. Figure 1 presents the evolution of $\bar{\gamma}$ for an on-axis emitter for a wide range of diameters, showing that $\bar{\gamma}<0.075$ for $d / \lambda<0.29$.

Self-assembled InAs QDs grown on GaAs are ideal emitters for exploiting the appealing properties of photonic nanowires for SE control. At cryogenic temperatures, they typically display a nearly perfect radiative yield. Moreover, the dipole associated to their fundamental optical transition is perpendicular to the growth axis [18]. Defining wires along this axis simultaneously guarantees an optimal coupling to the guided mode and an efficient screening of the other modes $[13,14]$. To provide direct experimental evidence of the inhibition of SE into the continuum of radiative modes, we propose utilizing the fast deconfinement of $\mathrm{HE}_{11}$ as $d / \lambda$ is reduced; in an infinitely long photonic nanowire, Fig. 1 shows that $\bar{\gamma}$ dominates the total SE rate for $d / \lambda<0.17$.

The photonic wires under study are sketched in the inset appearing in Fig. 4. To optimize the collection of light in the far field, a modal mirror is integrated below the wire and the upper tip features a conical taper that reduces the divergence of the output beam. A self-assembled QD layer, with a $\sim 300 \mu \mathrm{m}^{-2}$ areal density, is located $80 \mathrm{~nm}$ above the mirror (11-nm-thick $\mathrm{SiO}_{2}$ spacer and 250-nm-thick gold layer). The ensemble luminescence peaks at $920 \mathrm{~nm}$, with a $50 \mathrm{~nm}$ inhomogeneous broadening. Thus, a 200-nm-diameter wire typically contains 10 randomly located QDs. To allow for systematic studies, a series of photonic wires with diameters varying by a fine 20-nm step were fabricated. The actual wire diameter at the dot location was measured with a \pm 10 -nm accuracy by using a scanning electron microscope. More details on the fabrication process can be found in Ref. [14].

Time-resolved microphotoluminescence (TRPL) experiments are carried out at cryogenic temperature $(5 \mathrm{~K})$ in a helium-flow cryostat. Carrier injection in the QDs is provided by a $200-\mathrm{fs}$ pulsed Ti:sapphire laser, with a repetition rate of $76 \mathrm{MHz}$. This laser beam, tuned at $820 \mathrm{~nm}$, is focused down to a 1.5 - $\mu$ m-diameter spot on the sample with a microscope objective (N.A. $=0.75$ ), which also collects the luminescence signal. The luminescence of the exciton $(X)$ line associated with a single dot is then

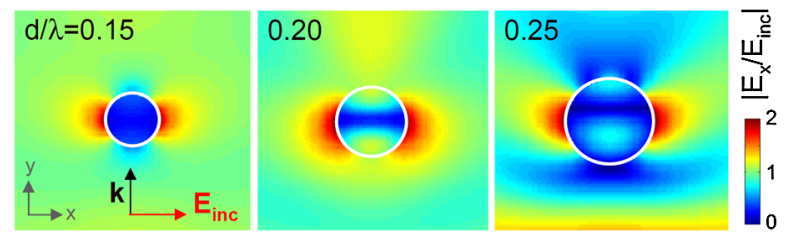

FIG. 2 (color online). Screening of an incident plane wave by photonic nanowires with various diameters. The figures are a color map of $\left|E_{x} / E_{\text {inc }}\right|$, i.e., the amplitude of the electrical field along $x$ normalized to the amplitude of the incident field. 
spectrally filtered by a $310-\mathrm{mm}$ focal length monochromator and detected by a silicon avalanche photodiode. The instrument response of the setup, measured with the excitation laser, presents a 300-ps FWHM associated with the time jitter of the avalanche photodiode. To provide a clean measurement of the $X$ decay time, measurements are conducted at low pumping power, well below the dot saturation.

Figure 3 shows the TRPL decay of 2 dots (named QD1 and QD2, red curves) embedded in wires with $d / \lambda=$ $0.13 \pm 0.01$. The ensemble PL decay of the as-grown sample (QDs $80 \mathrm{~nm}$ below the air-semiconductor interface) serves as a reference. The data clearly show a dramatic slowdown of the SE rate $\Gamma$. For a quantitative analysis, the data are fitted to a monoexponential decay $\propto \exp (-\Gamma t)$ convolved with the measured instrument response of the setup (including the dark count level of the detector). The as-grown sample decay is then $0.59 \mathrm{~ns}^{-1}$, which corresponds to $\Gamma_{0}=0.71 \mathrm{~ns}^{-1}$, after correction of the interference effect due to the air-semiconductor interface [19]. In stark contrast, $\Gamma=0.045 \mathrm{~ns}^{-1}\left(0.068 \mathrm{~ns}^{-1}\right)$ for QD1 (QD2), leading to an inhibition factor $\bar{\Gamma}^{-1}=16$ (10). Similar performances are only achieved in state-of-theart, 2D-photonic crystals [5], which are much more complex structures.

We now focus on larger wires to investigate the coupling to $\mathrm{HE}_{11}$. Within the simple Fabry-Perot model developed in Ref. [13], the reflection at the wire ends modifies $\bar{\Gamma}_{m}$ and leaves $\bar{\gamma}$ unchanged. The conical taper suppresses the reflectivity of the top facet [20], and the reflection on the bottom mirror generates a standing-wave pattern between

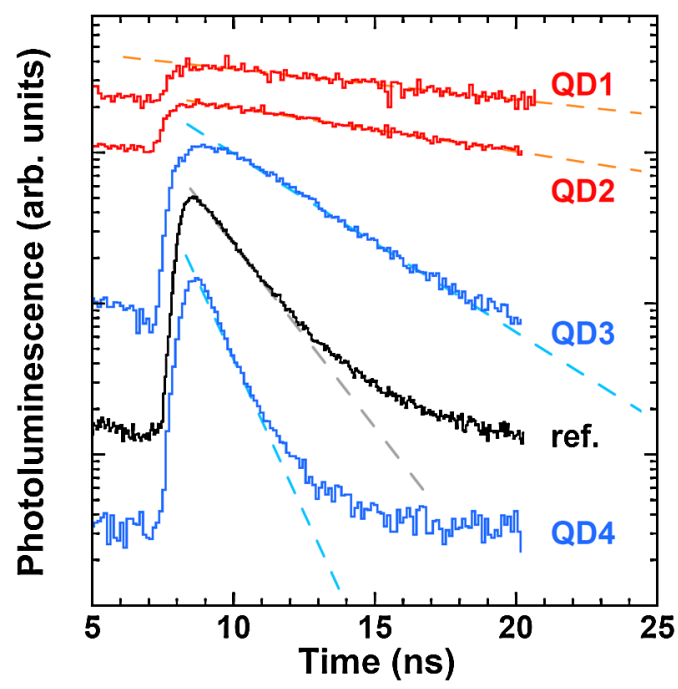

FIG. 3 (color online). TRPL decay associated with the $X$ transition of single QDs embedded in photonic nanowires (logarithmic vertical scale). QD1 and QD2 (QD3 and QD4) are embedded in a wire with $d / \lambda=0.13 \pm 0.01(0.25 \pm 0.01)$. The trace Ref. is associated to the as-grown reference sample. The lines are guides for the eye, and the decay rates are extracted from the fitting procedure described in the text. the emitter and the mirror. Locating the emitter on a longitudinal antinode of the field enhances $\bar{\Gamma}_{m}$ by a factor $\left(1+\left|r_{m}\right|\right)$, where $\left|r_{m}\right|$ is the modal reflectivity of the mirror. In the sample, the emitters are precisely located on the first antinode for $d / \lambda=0.258$ [21].

The blue curves in Fig. 3 show the TRPL traces for two dots embedded in a photonic nanowire with $d / \lambda=0.25 \pm$ 0.01 . The fastest dot (QD4) exhibits $\Gamma=1.1 \mathrm{~ns}^{-1}$. The corresponding SE enhancement factor, $\bar{\Gamma}=1.5$, is close to the theoretical maximum value of 1.65 [13], expected for an on-axis emitter. This result also constitutes evidence of the high modal reflectivity of the integrated mirror. As the emitter-axis distance $x$ increases, $\bar{\Gamma}_{m}$ decreases, at a faster pace than $\bar{\gamma}$ increases. As a consequence, some dots, such as QD3, exhibit a normalized SE rate as low as $\bar{\Gamma}=0.39$.

To provide a comprehensive picture of $\mathrm{SE}$ in photonic wires, we plot in Fig. 4 the measured normalized SE rate $\bar{\Gamma}$ of 60 QDs embedded in wires with various diameters. To support the analysis, we have calculated the maximum and minimum $\bar{\Gamma}$, shown with solid curves [22]. Two regimes can be distinguished on this plot. Below $d / \lambda=0.16$, the SE is strongly inhibited and SE rates are quite uniform: $\bar{\Gamma}$ is dominated by $\bar{\gamma}$, which is relatively independent of the QD location in the wire section [see Fig. 2]. QD1 and 2 belong to this limit. Above $d / \lambda=0.16, \bar{\Gamma}$ increases and shows stronger variations associated to the spatial variations of $\bar{\Gamma}_{m}$ and $\bar{\gamma}$. The global agreement between theory and experiments demonstrates a rather good understanding of SE dynamics in photonic nanowires.

This demonstration of a very strong inhibition of SE into the radiative modes, combined with a moderate enhancement of SE into the guided mode, confirm the potential of photonic nanowires to achieve $\beta>0.9$ for on-axis emitters. Next, we address the important question of the

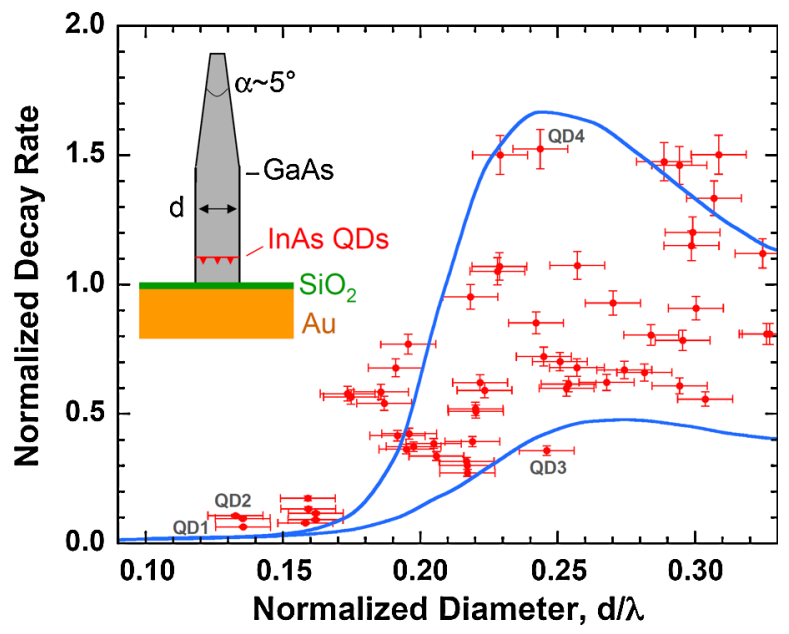

FIG. 4 (color online). Compilation of the measured decay rates of 60 single QDs in photonic nanowires with various diameters. The data are bounded by the calculated maximum and minimum total SE rates (solid lines), obtained by varying the lateral position of the emitter in the wire. Inset: Structure of the sample. 


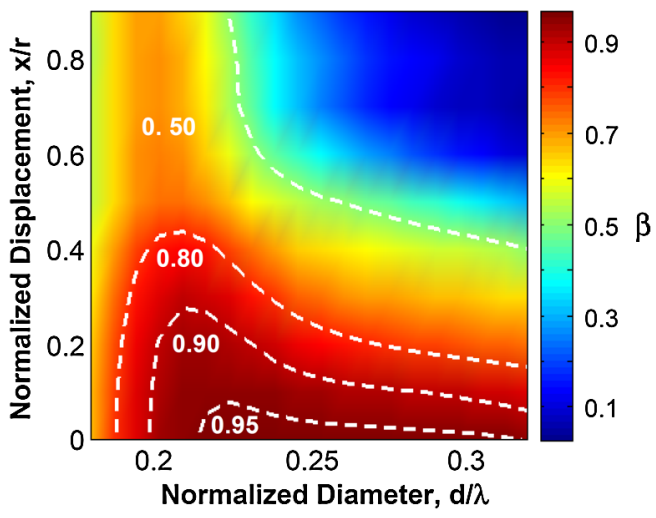

FIG. 5 (color online). Fully vectorial calculation of $\beta$ in the $(x / r ; d / \lambda)$ parameter space, for the finite-length photonic nanowires studied in this work.

robustness of $\beta$ for off-axis emitters. Figure 5 presents the calculated evolution of $\beta$ in the $(x / r ; d / \lambda)$ parameter space, for an emitter having an in-plane, isotropic dipole. For $d / \lambda \sim 0.21$, one randomly located QD out of 15 experiences $\beta>0.9$, and one QD out of 5 experiences $\beta>0.8$. Considering the areal density of the QDs, a wire containing a dot with $\beta>0.9$ is typically found after 2 tries. Contrary to cavity-based SE control, the spectral and spatial distribution of self-assembled QDs is not a serious limitation to achieve high $\beta$ 's in a reasonable fraction of the devices. Core-shell growth offers a promising, alternative route to fabricate photonic nanowires embedding an exactly on-axis, single emitter. In that case, a thin wire featuring a longitudinal heterostructure first realizes the QD, and a subsequent lateral overgrowth provides the optimal diameter for the photonic confinement [23,24].

Interestingly enough, the strong inhibition of the radiative recombination that occurs for small diameters can be used to determine the radiative efficiency $\eta$ of the emitter. Experimentally, $\Gamma=45 \mu \mathrm{s}^{-1}$ for QD1 $\left(68 \mu \mathrm{s}^{-1}\right.$ for QD2), which is larger than the theoretical $23 \mu \mathrm{s}^{-1} \mathrm{SE}$ rate. This points toward a nonradiative recombination channel, with a rate of $22 \mu \mathrm{s}^{-1}\left(45 \mu \mathrm{s}^{-1}\right)$. Using the $0.71 \mathrm{~ns}^{-1}$ reference decay rate results in $\eta=0.97$ (0.94). This analysis provides a confirmation of the high radiative yield of QDs and shows that $\eta$ is preserved in nanowires with a diameter as low as $120 \mathrm{~nm}$. For smaller wires, no exploitable signal was found, likely because of the onset of surface nonradiative recombinations [19]. Because of these, let us mention that measuring $\eta$ in nanowires provides a lower bound for the efficiency of QDs embedded in bulk GaAs.

In conclusion, we have shown that photonic nanowires provide a very strong inhibition of SE into the continuum of radiative modes that lead to a very efficient funneling of SE into the guided mode. These structures are thus appealing candidates for the realization of efficient quantum light sources such as sources of single photons or entangled-photon pairs. Moreover, the marked inhibition of the total SE rate obtained for "small" wires provides a convenient tool to measure the radiative yield of the embedded emitters.

The authors thank M. Bazin for the epitaxial growth of the sample. Financial support from the IST-FET European project QPhoton, the "Nanosciences aux limites de la Nanoélectronique" Foundation, and the NanoEPR project of the 2006 NanoSci-ERA European program are acknowledged. Part of the sample fabrication was performed in the "Plateforme technologique amont" and CEA LETI MINATEC/DOPT clean rooms.

*joel.bleuse@cea.fr

†julien.claudon@cea.fr

[1] T. Baba et al., IEEE J. Quantum Electron. 27, 1347 (1991).

[2] For a review, see J. M. Gérard, Top. Appl. Phys. 90, 269 (2003); K. J. Vahala, Nature (London) 424, 839 (2003).

[3] E. Yablonovitch, Phys. Rev. Lett. 58, 2059 (1987).

[4] P. Lodahl et al., Nature (London) 430, 654 (2004).

[5] S. Noda, M. Fujita, and T. Asano, Nat. Photon. 1, 449 (2007).

[6] G. Lecamp, P. Lalanne, and J. P. Hugonin, Phys. Rev. Lett. 99, 023902 (2007); V.S. C. Manga Rao and S. Hughes, Phys. Rev. B 75, 205437 (2007).

[7] T. Lund-Hansen et al., Phys. Rev. Lett. 101, 113903 (2008).

[8] M. Bayer et al., Phys. Rev. Lett. 86, 3168 (2001).

[9] D. Englund et al., Phys. Rev. Lett. 95, 013904 (2005).

[10] J. P. Zhang et al., Phys. Rev. Lett. 75, 2678 (1995). Recent calculations show that in this geometry $\beta$ peaks at a value of 0.65 ; see details in G. Lecamp, P. Lalanne, and J.P. Hugonin, Proc. SPIE Int. Soc. Opt. Eng. 6195, 61950E (2006).

[11] V. V. Klimov and M. Ducloy, Phys. Rev. A 69, 013812 (2004).

[12] A. V. Maslov, M. I. Bakunov, and C.Z. Ning, J. Appl. Phys. 99, 024314 (2006).

[13] I. Friedler et al., Opt. Express 17, 2095 (2009).

[14] J. Claudon et al., Nat. Photon. 4, 174 (2010).

[15] T. M. Babinec et al., Nature Nanotech. 5, 195 (2010).

[16] J. P. Hugonin and P. Lalanne, J. Opt. Soc. Am. A 22, 1844 (2005).

[17] B.Z. Katsenelenbaum, Zh. Tekh. Fiz. XIX, 1182 (1949).

[18] S. Cortez et al., Phys. Rev. B 63, 233306 (2001).

[19] J. Johansen et al., Phys. Rev. B 77, 073303 (2008).

[20] N. Gregersen et al., Opt. Lett. 33, 1693 (2008).

[21] The interference pattern, and thus the location of the longitudinal antinodes, depends on the effective index of the guided mode, which in turn depends on $d / \lambda$.

[22] The emitter position is varied from $x=0$ to $x=7 r / 8$. The SE rate is obtained by averaging the SE rates associated to radial and orthoradial polarizations.

[23] M. Tchernycheva et al., Nano Lett. 7, 1500 (2007).

[24] J. Heinrich et al., Appl. Phys. Lett. 96, 211117 (2010). 